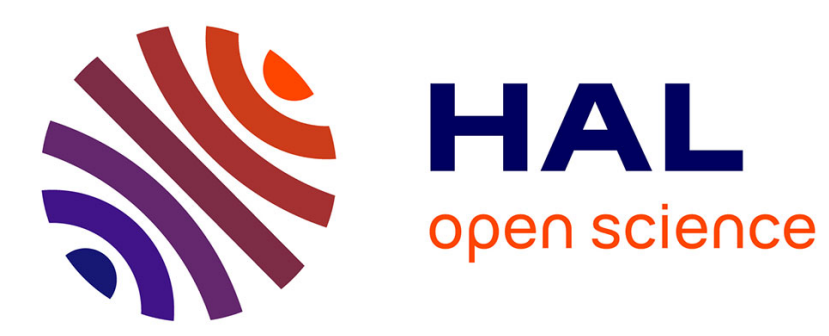

\title{
Les combattants irlandais et britanniques face au soulèvement de Dublin du 24 avril 1916 \\ Emmanuel Destenay
}

\section{To cite this version:}

Emmanuel Destenay. Les combattants irlandais et britanniques face au soulèvement de Dublin du 24 avril 1916. Guerres mondiales et conflits contemporains, 2014, 256 (4), pp.131-147. 10.3917/gmcc.256.0131 . hal-03416561

\section{HAL Id: hal-03416561 \\ https://hal.sorbonne-universite.fr/hal-03416561}

Submitted on 5 Nov 2021

HAL is a multi-disciplinary open access archive for the deposit and dissemination of scientific research documents, whether they are published or not. The documents may come from teaching and research institutions in France or abroad, or from public or private research centers.
L'archive ouverte pluridisciplinaire HAL, est destinée au dépôt et à la diffusion de documents scientifiques de niveau recherche, publiés ou non, émanant des établissements d'enseignement et de recherche français ou étrangers, des laboratoires publics ou privés. 


\section{LES COMBATTANTS IRLANDAIS FACE AU SOULÈVEMENT DE DUBLIN DU 24 AVRIL 1916}

S'intéresser aux expériences de guerre des combattants irlandais de la Première Guerre mondiale c'est mesurer l'impact du soulèvement du 24 avril 1916 de Dublin sur les unités irlandaises en faisant entrer dans toute sa complexité l'insurrection dans l'historiographie de leur participation au Premier Conflit mondial.

Les études portant respectivement sur les trois divisions irlandaises ${ }^{1}$ et sur les régiments irlandais du Corps Expéditionnaire britannique ${ }^{2}$ mentionnent effectivement cette révolte. Cependant, les historiens n'ont pas étudié les conséquences de l'insurrection sur le moral des engagés volontaires et des militaires de métier irlandais. Jusqu'alors, les travaux universitaires traitaient uniquement de la réaction des officiers irlandais au front suite au soulèvement du 24 avril 1916 sans mesurer le retentissement de la révolte auprès des soldats du rang ${ }^{3}$. Par ailleurs, les études se limitaient au champ irlandais et ne traitaient pas de l'influence de la rébellion sur les autres combattants de l'armée britannique.

Étudier le comportement des troupes irlandaises après l'insurrection d'avril 1916 s'avère une entreprise difficile. Les témoignages écrits sont peu nombreux et, surtout, généralement brefs. La plupart du temps, les récits de guerre des combattants irlandais n'évoquent pas l'insurrection. L'absence d'une presse des tranchées constitue une difficulté supplémentaire pour qui veut essayer d'en mesurer l'impact. En effet, pendant toute la durée du conflit, il semble qu'il n'y ait eu qu'un seul journal de tranchées, The Incinerator, journal du $14^{\text {ème }}$ bataillon des Royal Irish Rifles de la $36^{\text {ème }}$ division d'Ulster, qui n'a paru que deux fois, en mai 1916 et en juin de la même année. En l'absence de ces sources de renseignements, il convient de se tourner vers d'autres documents jusqu'alors peu sollicités pour apprécier la réaction des unités irlandaises du Corps Expéditionnaire britannique et des engagés volontaires des trois divisions irlandaises.

En croisant les sources inédites disponibles à l'Imperial War Museum de Londres, à la Bibliothèque municipale de Dublin et à la Bibliothèque universitaire de Leeds, cet article propose d'étudier les réactions des soldats et officiers irlandais de l'armée britannique après l'insurrection du 24 avril 1916. En effet, en mettant en lumière la diversité des comportements au sein des unités irlandaises, cette recherche permet de mieux cerner la loyauté des combattants du Corps Expéditionnaire britannique et des trois divisions irlandaises $\left(10^{\mathrm{ème}}\right.$,

\footnotetext{
${ }^{1}$ Bryan Cooper, The $10^{\text {th }}$ (Irish) Division at Gallipoli (1918), Dublin, Irish Academic Press, 1995, 156 p; Cyril Falls, The History of the $36^{\text {th }}$ (Ulster) Division, London, Constable, 1922, 359 p; Terence Denman, Ireland's Unknown Soldiers: the 16 ${ }^{\text {th }}$ Division in the Great War, Dublin, Irish Academic Press, 1992, 209 p.

${ }^{2}$ Henry Harris, The Irish Regiments in the First World War, Cork, Mercier Press, 1968, 229 p; Tom Johnstone, Orange, Green and Khaki: Story of the Irish Regiments in the Great War, 1914-18, Dublin, Gill \& Macmillan, 1992, $498 \mathrm{p}$.

${ }^{3}$ Jane Leonard, 'The Reaction of Irish Officers in the British Army to the Easter Rising of 1916', in Cecil Hugh \& Peter Liddle (eds.), Facing Armageddon: The First World War Experienced, London, L. Cooper, 1996, pp. 256-268.
} 
$16^{\text {ème }}$ et $36^{\text {ème }}$ divisions) levées pour l'effort de guerre ${ }^{4}$. Notre étude ne se limite cependant pas au seul cas irlandais; elle aborde également les conséquences de l'insurrection sur le moral d'autres unités de l'armée britannique. Élargissant ainsi le champ d'étude, ce travail permet de compléter les recherches sur le moral des troupes britanniques pendant le Premier Conflit mondial 5 . L'analyse des répercussions de l'insurrection sur le moral des autres combattants britanniques amène donc à ne plus considérer le soulèvement de Dublin comme un événement isolé qui n'affecterait que les troupes irlandaises au front. Elle montre aussi en quoi l'historiographie de la Première Guerre mondiale gagne à ne plus envisager les nouvelles de l'arrière uniquement comme des « béquille[s] déterminante[s $]^{6}$ » fortifiant le moral des combattants.

\section{Défier l’Empire britannique pendant le Premier Conflit mondial}

Le 4 août 1914, le Royaume-Uni déclare la guerre à l'Allemagne. La Grande-Bretagne entre résolument dans le conflit, mais l'Irlande se trouve idéologiquement divisée entre les partisans d'un État autonome et les unionistes majoritairement implantés dans le nord du pays. Alors que l'île fait toujours partie du Royaume-Uni et que les parlementaires nationalistes siégeant à Westminster militent depuis plusieurs années pour l'autonomie du pays sur le modèle des dominions britanniques, les députés unionistes, fidèles à la Couronne, rejettent catégoriquement une éventuelle autorité émanant de Dublin.

Dès août 1914, plusieurs dizaines de milliers de nationalistes irlandais, certains que Londres leur garantirait l'établissement d'un État libre « une fois que les armes se seraient tues $^{7} »$, s'engagent dans l'armée britannique. Alors que les fervents partisans d'une autonomie irlandaise participent au conflit pour obtenir l'avènement d'un état autonome, les députés unionistes lancent un appel à tous les fidèles de la Couronne pour les inciter à partir défendre l'Empire britannique. Soutenir l'effort de guerre leur permet d'affirmer leur patriotisme et de démontrer leur loyauté envers la Couronne. C'est aussi le seul moyen pour eux de s'opposer à l'autonomie éventuelle de l'île en affirmant leur appartenance britannique.

Or, quelques nationalistes parmi plus intransigeants, espérant tirer profit de l'entrée en guerre du Royaume-Uni dans le Premier Conflit mondial, se réunissent le 9 septembre 1914 à Dublin et jurent d'ébranler l'Empire en déclarant l'indépendance de l'Irlande. Le lundi 24 avril 1916, une poignée de nationalistes irlandais déclenche une rébellion au cœur de la capitale. Après cinq jours d'affrontements, le soulèvement, connu sous le nom de «Pâques Sanglantes », aboutit à un échec ${ }^{8}$. Prêts à libérer le pays de la présence britannique, quelques

\footnotetext{
${ }^{4}$ Précisons qu'à ce stade de nos recherches nous n'avons pas eu accès à des témoignages de combattants de la $10^{\text {ème }}$ division irlandaise dans lesquels les hommes évoquent le soulèvement. Cette étude s'appuie ainsi exclusivement sur les écrits des militaires de métiers irlandais du Corps Expéditionnaire britannique et sur les récits de guerre des engagés volontaires des $16^{\text {ème }}$ et $36^{\text {ème }}$ divisions irlandaises.

5 John Fuller, Troops Moral and Popular Culture in the British and Dominion Armies, Oxford, Clarendon Press, 1990, 218 p; Gary Sheffield, Leadership in the Trenches: Officer-Man Relations, Morale and Discipline in the British Army in the Era of the First World War, London, Macmillan, 2000, 296 p; Timothy Bowman, The Irish regiments in the Great War-discipline and morale, Manchester, Manchester University Press, 2004, 237 p.

${ }^{6}$ François Cochet, Survivre au front, 1914-1918. Les poilus entre contrainte et consentement, Saint-Cloud, 14-18 Editions, 2005, p. 200.

${ }^{7}$ Tom Johnstone, Orange, Green and Khaki, op. cit., p. 9.

${ }^{8}$ Max Caulfield, The Easter Rebellion, Dublin, Gill \& Macmillan Ltd, 1995, 313 p; Tim Coogan, 1916: The Easter Rising, London, Phoenix, 2005, 195 p; Charles Townshend, Easter 1916: the Irish rebellion, London, Penguin Books, 2005, 360 p.
} 
680 rebelles occupent le centre-ville de Dublin forçant les autorités britanniques à mobiliser les troupes stationnées sur tout le territoire irlandais ${ }^{9}$. Mises en difficultés par les insurgés, les autorités britanniques doivent recourir à des renforts dès le premier jour des combats ${ }^{10}$. Les 500 soldats britanniques disponibles le lundi de Pâques ne suffisent pas à déloger les rebelles. Une poignée d'insurgés parvient à défier le gouvernement britannique qui se voit alors dans l'obligation de mobiliser des renforts pour écraser la rébellion et éviter qu'elle ne s'étende à l'ensemble du pays. Le lundi 25 avril, les autorités rappellent ainsi 2500 hommes du Curragh, le plus important centre d'entraînement militaire en Irlande. Le lendemain, 1000 soldats de Belfast et trois mitrailleuses en provenance d'Athlone, à 130 kilomètres à l'ouest de Dublin, arrivent également dans la capitale ${ }^{11}$.

Des renforts arrivent aussi de Grande-Bretagne. Le gouvernement britannique mobilise plusieurs unités anglaises pour sécuriser le territoire irlandais. C'est ainsi que le $7^{\text {ème }}$ bataillon des Sherwood Forester quitte ses casernes en Angleterre pour aller «écraser la révolte $^{12}$ ». L'unité embarque à Liverpool le 25 avril et livre ses premiers combats en Irlande $^{13}$. Les soldats d'un bataillon du Lincolnshire Regiment quittent eux aussi la GrandeBretagne pour l'île où ils essuient leurs premiers tirs ${ }^{14}$. À leur arrivée dans la capitale, les soldats se réjouissent de l'accueil chaleureux de la population. Un comité de Dublinois s'organise pour ravitailler « les soldats britanniques pendant le soulèvement de Pâques ${ }^{15}$ ». De jeunes femmes s'empressent d'aller au-devant des troupes qui marchent vers la capitale et leur offrent « des cigarettes, du lait et de l'eau ${ }^{16} »$. D'autres se hâtent de leur apporter « du thé et des casse-croûtes ${ }^{17}$ ». Les soldats britanniques qui arrivent de Liverpool «affamés ${ }^{18}$ »se réjouissent de cette marque d'attention.

\section{Les résistances à l'autorité britannique des engagés volontaires irlandais}

Lors du soulèvement, la plupart des 2400 soldats britanniques en poste dans les casernes militaires de Dublin sont de nationalité irlandaise ${ }^{19}$. C'est le cas de George Francis Hare et de ses quatre frères lesquels, engagés en 1914 dans l'armée britannique, partent mater la rébellion irlandaise avec leur bataillon des Royal Dublin Fusiliers ${ }^{20}$. Le sous-lieutenant Jameson, qui s'attendait à connaître sa première expérience de guerre sur le continent, essuie ses premiers tirs lors de l'insurrection. Avec le $5^{\text {ème }}$ bataillon des Leinster, il quitte les casernes militaires du Curragh à minuit le 24 avril 1916 et fait route vers Dublin. Les engagés volontaires irlandais du bataillon des Leinster livrent leurs premiers combats contre les

\footnotetext{
${ }^{9}$ Royal Commission on the Rebellion in Ireland: Minutes of Evidence and Documents Relative to the Sinn Féin Movement, HMSO, Westminster, p. 9.

${ }^{10}$ Bibliothèque Nationale d'Irlande (désormais NLI), MS. 34,256A, « Journal intime de Mary Martin (janviermai 1916)», 26 avril 1916.

${ }^{11}$ Royal Commission on the Rebellion in Ireland: Minutes of Evidence and Documents Relative to the Sinn Féin Movement, HMSO, Westminster, p. 9.

${ }^{12}$ Bibliothèque universitaire de Leeds, Collection Liddle, GS 0572, lettre du capitaine Foster à ses parents, 21 mai 1916 ; GS 1387, lettre du capitaine Round à sa tante, 11 mai 1916.

${ }^{13}$ Ibidem, cassette 1349, entretien de Rogers, $7^{\text {ème }}$ bataillon des Sherwood Foresters, avec Peter Liddle, 2 février 1998.

${ }^{14}$ Ibidem, Collection, Domestic Front : Ireland, dossier 15, entretien de Lingwood avec Peter Liddle, janvier 1971 .

${ }^{15}$ NLI, MS. 34,256A, « Journal intime de Mary Martin (janvier-mai 1916) », 5 mai 1916.

${ }^{16}$ Archives de la Bibliothèque municipale de Dublin (désormais DCLA), Archives des Royal Dublin Fusiliers,

«Collection Monica Roberts », Volume VIII, journal intime de Monica Roberts, p. 11.

${ }^{17}$ LUL, Collection Liddle, GS 0403, G.H. Crowe, « Recollections », p. 6.

${ }^{18}$ Ibidem, Domestic Front : Ireland, dossier 15, entretien de F. Lingwood avec Peter Liddle, janvier 1971.

${ }^{19}$ Jeff Kildea, Anzacs and Ireland, Cork, Cork University Press, 2007, p. 75.

${ }^{20}$ Idem.
} 
rebelles dans la capitale irlandaise. Ils partent défaire les insurgés irlandais alors que leur engagement dans l'armée britannique avait été motivé par le combat contre les Allemands.

Alors que des engagés volontaires participent à la répression du soulèvement, d'autres recrues irlandaises refusent de tirer sur les rebelles. Ainsi, Charles Duff, rentrant de permission de Fermanagh, reçoit en même temps que ses compagnons l'ordre de se présenter aux casernes pour aller écraser l'insurrection. Lui et une petite vingtaine de volontaires protestent avec indignation ; ils rappellent s'être engagés pour combattre les Allemands et non pour mater une rébellion et tirer sur leurs concitoyens. Leur officier décide alors de les enfermer pour la nuit en guise de punition mais ne rédige pas de rapport ${ }^{21}$. Aucune sanction n'est prise. Les insoumis ne passent même pas en cour martiale pour désobéissance. L'attitude du commandant mérite réflexion. Alors même que la capitale irlandaise est en proie à de violents combats, comment expliquer la clémence de l'officier face à cet acte d'insubordination? Interrogé sur les raisons de son silence, le gradé répond que « ce ne serait pas faire preuve de bon sens que de confronter des rebelles irlandais à des Irlandais qui ne voulaient pas combattre leurs compatriotes et qui pourraient même éprouver de la compassion pour eux ${ }^{22}$ ». L'utilisation des mots «compatriotes » et «compassion » suggèrent que les rebelles de 1916 et les engagés de 1914-1918 appartiennent à un même peuple, à une même «nation ». L'explication du major laisse aussi supposer une certaine crainte. C'est aussi une manière de ne pas prendre de risques, de ne pas provoquer de mutinerie au sein du bataillon. Émanant de celui qui incarne le commandement britannique, la décision du major apparaît d'autant plus étonnante et inappropriée. Les engagés agissent comme des partisans qui refusent d'exécuter un ordre pour ne pas trahir leurs convictions politiques. En convalescence dans sa ville natale lors de l'insurrection, George McElroy tient lui aussi tête à son supérieur lorsque ce dernier le somme de rejoindre son unité pour écraser la rébellion. Engagé volontaire en août 1914, le soldat du Royal Irish Regiment désobéit. En "guise de punition $^{23}$ », son supérieur l'envoie rejoindre une garnison dans le sud du pays. L'officier préfère ici éloigner le soldat plutôt que de le traduire en cour martial pour indiscipline. S'engager dans l'armée britannique pendant le Premier Conflit mondial n'implique donc pas une loyauté inconditionnelle envers le Couronne britannique.

Si certains soldats irlandais refusent de tirer sur leurs concitoyens, d'autres vont plus loin, en décidant de rejoindre les rebelles. Le commandant Thomas Byrne, capitaine d'un bataillon d'insurgés lors de l'insurrection, certifie que plusieurs jeunes recrues des Royal Dublin Fusiliers prennent part aux combats auprès des révolutionnaires. Les «transfuges » se trouvent avec le commandant Byrne retranchés dans une maison à l'angle de Liffey Street quelques jours avant la fin des combats ${ }^{24}$. S'opère ainsi un transfert de loyauté. Les recrues profitent du soulèvement pour renier l'uniforme britannique et combattre auprès des insurgés.

Les témoignages de Charles Duff et de Thomas Byrne restent à ce jour les seuls dont nous disposions. Leurs récits suggèrent que l'engagement dans l'armée britannique pendant le Premier Conflit mondial n'implique pas nécessairement un sentiment de loyauté absolue envers la Couronne. En effet, si $134202^{25}$ volontaires irlandais s'engagent dans l'armée britannique pendant toute la durée du conflit, tous ne nourrissent pas des aspirations unionistes. Certaines recrues s'engagent en espérant que «cette démonstration d'amitié et de

\footnotetext{
${ }^{21}$ Charles Duff, Six Days To Shake An Empire; events and factors behind the Irish Rebellion of 1916, London, J. M. Dent, 1966, p. 105.

${ }^{22}$ Idem.

${ }^{23} \mathrm{http} / / /$ www.firstworldwar.com/bio/mcelroy.htm. Site consulté en mai 2014.

${ }^{24}$ Archives Militaires d'Irlande (Cathal Brugha), BMH. WS 0564, témoignage de Thomas Byrne, capitaine des Irish Volunteers, commandant-en-chef de la $1^{\text {ère }}$ brigade républicaine de Dublin, p. 22.

${ }^{25}$ Statistics of Military Effort of the British Empire during the Great War, London, HMSO, Westminster, 1922 , p. 363. À cette époque, la population totale de l'île est estimée à 4374500 .
} 
loyauté [envers la couronne] ${ }^{26} »$ permettrait l'établissement d'un État libre une fois la guerre terminée. D'autres rejoignent les rangs de l'Armée britannique pour « voir comment était la guerre, avoir un fusil [et] voir de nouveaux pays $^{27} \gg$. Ainsi, si les recrues acceptent d'aller se battre contre l'Allemagne, certains se révoltent à l'idée de devoir réprimer une insurrection nationale. Accepter de se porter volontaire, c'est accepter de combattre les Allemands. Cela ne suggère pas nécessairement une remise en cause de ses convictions politiques et idéologiques. Des fidèles partisans d'une nation irlandaise indépendante n'hésitent pas à s'allier aux rebelles pour tenter de défaire l'armée dans laquelle ils se sont engagés. Les «transfuges », engagés volontaires dans l'armée britannique, trahissent ainsi l'uniforme britannique.

\section{Incompréhension et indignation des militaires irlandais de métier}

Qu'en est-il alors des soldats irlandais au front ? Des combattants refusent-ils d'obéir à leurs supérieurs et déposent-ils les armes ?

C'est par les quotidiens français que certaines unités de l'armée britanniques prennent éventuellement connaissance des nouvelles de la rébellion dès le 26 avril ${ }^{28}$. Alors qu'il se trouve dans la Somme, le $2^{\text {ème }}$ bataillon des Royal Dublin Fusiliers apprend les nouvelles venues d'Irlande «d'après les journaux ${ }^{29}$ ». La correspondance constitue aussi une source d'informations non négligeable pour les combattants. C'est notamment grâce aux lettres de leurs marraines de guerre que plusieurs soldats irlandais du Corps Expéditionnaire britannique sont informés des «émeutes de Dublin ${ }^{30}$ ». Les récits détaillés des permissionnaires revenus au front permettent également aux unités de mesurer la gravité de la situation ${ }^{31}$. Les nouvelles parviennent aussi aux unités britanniques par «TSF». C'est de cette manière que quelques soldats et officiers stationnés en Égypte à cette période comprennent la sévérité de la révolte ${ }^{32}$.

Au sein du Corps Expéditionnaire britannique, les unités irlandaises hésitent entre déception et amertume. Joseph Clarke, du $2^{\text {ème }}$ bataillon des Royal Dublin Fusiliers, souligne qu'il n'y a pas «de troupes qui ne soient plus désolées d'apprendre les nouvelles du soulèvement que les troupes irlandaises ${ }^{33} »$. C'est aussi avec désolation que les camarades écoutent les nouvelles de l'insurrection qui « couvre le pays de honte ${ }^{34}$ ». L'aumônier de cette unité confie lui aussi que « rien n'a autant attristé les [soldats du $2^{\text {ème }}$ bataillon des Royal Irish Rifles] ${ }^{35} \gg$ que les nouvelles du soulèvement. Le capitaine Faithfull, du $1^{\text {er }}$ bataillon des Royal Irish Regiment, qualifie le soulèvement de «scandaleux ${ }^{36}$ » et ne parvient pas à comprendre comment le gouvernement britannique a pu laisser faire. "Triste événement ${ }^{37}$ »,

\footnotetext{
${ }^{26}$ Tom Johnstone, Orange, Green and Khaki, op. cit., p. 9.

${ }^{27}$ Tom Barry, Guerilla Days, Dublin, Dublin Irish Press, 1949, p. 2.

${ }^{28}$ LUL, Collection Liddle, GS 0644, lettre du sous-lieutenant Goodwin à sa mère, 26 avril 1916.

${ }^{29} \mathrm{http} / / /$ www.europeana1914-1918.eu/fr/contributions/3632. Site consulté en janvier 2014.

${ }^{30}$ DCLA, Archives des Royal Dublin Fusiliers, « Collection Monica Roberts », vol. II, RDFA/001/14, lettre de Christopher Fox à Monica Roberts, 12 mai 1916.

${ }^{31}$ Ibidem, vol. I, RDFA/001/01, lettre de Joseph Clarke à Monica Roberts, 11 mai 1916.

32 Bernard Carey, Leaves From The Diary of A Catholic Chaplain in The Great War, Pittsburg, Duquesne University, 1920, p. 45.

${ }^{33}$ DCLA, Archives des Royal Dublin Fusiliers, « Collection Monica Roberts », vol. I, RDFA/001/01, lettre de Joseph Clarke à Monica Roberts, 11 mai 1916.

${ }^{34}$ Ibidem, vol. II, RDFA/001/14, lettre de Christopher Fox à Monica Roberts, 12 mai 1916.

35 Archives des Jésuites de Dublin (désormais JAD), CHP 1/25, correspondance du Père Henry Vincent Gill, aumônier dans le $2^{\text {ème }}$ bataillon des Royal Irish Rifles, lettre à l'archevêché, 5 mai 1916.

${ }^{36}$ LUL, Collection Liddle, GS 0539, lettre du capitaine Faithfull à ses parents, 24 mai 1916.

${ }^{37}$ DCLA, Archives des Royal Dublin Fusiliers, «Collection Monica Roberts », vol. II, RDFA/001/15, lettre de Thomas Finn à Monica Roberts, 13 juin 1916.
} 
«incompréhensible ${ }^{38} »$, ces termes qualifient le soulèvement mais, au-delà même de l'incompréhension qui les domine, les militaires de métier irlandais s'inquiètent pour leurs proches.

Alors qu'il se trouve dans la Somme avec son unité, Christopher Fox craint que «ces lâches assassins ne tuent les mères, les pères [peut-être même] les femmes et les enfants des pauvres gars qui se trouvent ici et qui combattent pour leur pays ${ }^{39} »$. Au sentiment insoutenable de la trahison se mêle l'angoisse. S'il est déjà suffisamment difficile de tenir au front, les nouvelles de la rébellion tétanisent les combattants très vite préoccupés pour leurs familles. Beaucoup «s'inquiètent pour la sécurité de leurs proches ${ }^{40}$ » qui vivent non loin des rues où se tiennent les affrontements ${ }^{41}$. Sans nouvelles de leurs familles, ils confient leurs angoisses à leurs marraines de guerre. Ainsi, ne pas savoir, ne pas avoir de nouvelles rassurantes des leurs contribuent à fragiliser le moral des soldats dont la plupart viennent de la capitale et de ses alentours. Les témoignages des combattants des Royal Dublin Fusiliers suggèrent en effet qu'en l'absence de nouvelles rassurantes de leur famille les soldats imaginent le pire. Il leur faut tenir au front et, de plus, affronter éventuellement la mort d'un parent.

Quelques «pauvres gars ${ }^{42}$ » déplorent, impuissants, depuis les champs de bataille de la Somme, la perte d'un des leurs. Lors de l'insurrection de Dublin, 318 civils, victimes des tris croisés des rebelles et des militaires britanniques, trouvent la mort dans le centre-ville de la capitale $^{43}$. Les rebelles n'hésitent d'ailleurs pas à prendre "une femme ou un enfant comme bouclier $^{44} \gg$ pour se protéger des balles britanniques. L'annonce de la mort d'un proche terrasse les combattants. Les hommes s'effondrent en présence de leurs camarades du bataillon qui en viennent très vite à maudire les insurgés. Les mots de Christopher Fox rendent compte de leur ressentiment :

Quand je pense à eux mon sang ne fait qu'un tour. Je pense à ces pauvres gars qui se trouvent ici et qui combattent pour leur pays et dont la mère, le père, la femme ou les enfants ont été tués par ces lâches assassins ${ }^{45}$.

Certains ne reculent pas devant l'imprécation et «ne demandent pas mieux que de voir les rebelles envoyés ici, au front ${ }^{46} »$. À travers les correspondances et les mémoires des combattants, il devient évident qu'à partir du mois de mai 1916, les militaires de métier irlandais commencent à éprouver une haine profonde pour les meneurs de l'insurrection. Autre constat, ce ne sont plus les Allemands qui obsèdent les soldats mais les rebelles irlandais. Les insurgés de la Semaine Sainte se substituent ainsi à l'ennemi allemand. Ce sont désormais «ces porcs ${ }^{47}$ » que le sous-lieutenant Butler préférerait abattre «plutôt que les Huns $^{48}$ ». La frustration consume les combattants qui voudraient «pouvoir mettre la main sur

\footnotetext{
${ }^{38}$ LUL, Collection Liddle, GS 1039, James McRoberts, $14{ }^{\text {ème }}$ bataillon des Royal Irish Rifles (Young Citizen Volunteers), « Recollections, 1914-1918 », p. 165.

${ }^{39}$ DCLA, Archives des Royal Dublin Fusiliers, « Collection Monica Roberts », vol. II, RDFA/001/14, lettre de Christopher Fox à Monica Roberts, 31 mai 1916.

${ }^{40}$ Ibidem, vol. I, RDFA/001/01, lettre de Joseph Clarke à Monica Roberts, 22 avril 1916.

${ }^{41}$ Ibidem, vol. II, RDFA/001/14, lettre de Christopher Fox à Monica Roberts, 12 mai 1916.

${ }^{42}$ Ibidem, lettre de Christopher Fox à Monica Roberts, 31 mai 1916.

${ }^{43}$ Royal Commission on the Rebellion in Ireland: Minutes of Evidence and Documents Relative to the Sinn Féin Movement, HMSO, Westminster, 1916, p. 9.

${ }^{44}$ Imperial War Museum, 98/13/1, lettre du sous-lieutenant Anthony Jameson à sa mère, 2 mai 1916.

${ }^{45}$ DCLA, Archives des Royal Dublin Fusiliers, « Collection Monica Roberts », vol. II, RDFA/001/14, lettre de Christopher Fox à Monica Roberts, 31 mai 1916.

${ }^{46}$ Ibidem, vol. I, RDFA/001/01, lettre de Joseph Clarke à Monica Roberts, 11 mai 1916.

${ }^{47}$ LUL, Collection Liddle, GS 0253, sous-lieutenant J.H. Butler, Royal Irish Rifles, Royal Flying Corps, «Recollections, 1914-1919», p. 27.

${ }^{48}$ Idem.
} 
[les rebelles] $]^{49} \gg$ pour se défouler. Les hommes enragent de ne pas pouvoir « en attraper quelques-uns [pour] leur faire passer un mauvais quart d'heure ${ }^{50} »$. La violence avec laquelle s'expriment certains militaires de carrière irlandais du Corps Expéditionnaire britannique en France trahit aussi leur impuissance à l'idée de ne pas pouvoir réprimer eux-mêmes la rébellion et venger la mort de leurs proches tombés sous les balles des rebelles.

George Soper ne mâche pas ses mots et considère qu' « il faut abattre [les rebelles] sur le champ ${ }^{51}$ ». Originaire de Rathvilly, dans le comté de Carlow, le sous-lieutenant Butler souhaite que son unité quitte le front pour la capitale irlandaise afin d'aller « exterminer ces pauvres types $^{52} \gg$. Les gradés britanniques constatent aussi une forte réprobation de la part des combattants irlandais de leur unité. Le sous-lieutenant Marshall, du $7^{\text {ème }}$ bataillon du East Surrey Regiment, témoigne de la virulence des propos des soldats irlandais de son unité qui «ne demandent pas mieux que planter [les rebelles] avec leur baïonnette ${ }^{53} »$. Certains combattants du Corps Expéditionnaire britannique «prient même ${ }^{54}$ » pour que les rebelles soient exécutés.

Les unités irlandaises du Corps Expéditionnaire britannique crient à la trahison. La violence de leurs propos témoigne de leur impuissance face à une situation qui voit la sécurité de leurs proches menacée du fait de la rébellion. Rien n'est plus insupportable que de devoir défendre un territoire étranger sans même pouvoir porter secours à sa famille.

\section{Des combattants poignardés dans le dos}

Les engagés volontaires de la $36^{\text {ème }}$ division d'Ulster peuvent difficilement contenir leur colère et leur indignation. Le 29 avril 1916, le lieutenant Patrick Hemphill écrivait : " Je suppose qu'ils vont pendre tous les meneurs; c'est ce que méritent les traitres ${ }^{55}$. »Et d'ajouter : «Le mieux c'est qu'ils vont tous passer en cour martiale qui n'est pas coutumière de clémence ${ }^{56}$. » L'assurance que les meneurs de l'insurrection vont être pendus satisfait le lieutenant. Les engagés de la $36^{\text {ème }}$ division d'Ulster n'éprouvent aucune compassion pour les rebelles. Les soldats du $14^{\text {ème }}$ bataillon des Royal Irish Rifles vont même jusqu'à déclarer qu'en ce qui les concerne, ils auraient préféré « constater un peu moins de pitié pour (...) ces chiens d'insurgés qui profitent de l'absence des troupes [...] pour piller, se révolter, et assassiner une foule d'innocents ${ }^{57} \gg$. Certes, la dureté des propos semble compréhensible dans la mesure où ces soldats unionistes combattent pour l'Empire et le Roi. En effet, la $36^{\text {ème }}$ division d'Ulster, fer de lance de la politique unioniste irlandaise, fustige toute velléité d'indépendance irlandaise. Leur indignation est aussi dirigée contre la fourberie des insurgés qui fomentent une rébellion alors même que l'Empire britannique lutte contre l'armée allemande. Ce coup de poignard dans le dos mérite selon eux un châtiment exemplaire. C'est pourquoi ils jugent totalement insuffisante la réaction des autorités britanniques qui sur les

\footnotetext{
${ }^{49}$ DCLA, Archives des Royal Dublin Fusiliers, « Collection Monica Roberts », vol. II, RDFA/001/14, lettre de Christopher Fox à Monica Roberts, 19 avril 1916.

${ }^{50}$ Ibidem, vol. II, RDFA/001/14, lettre de Christopher Fox à Monica Roberts, 12 mai 1916.

${ }^{51}$ DCLA, Archives des Royal Dublin Fusiliers, « Collection Monica Roberts », vol. IV, RDFA/001/2, lettre de George Soper à Monica Roberts, 20 mai 1916.

${ }^{52}$ LUL, Collection Liddle, GS 0253, sous-lieutenant J.H. Butler, Royal Irish Rifles, Royal Flying Corps, « Recollections, 1914-1919», p. 27.

${ }^{53}$ Ibidem, GS 1056, lettre du sous-lieutenant R. B. Marshall à sa mère, 4 mai 1916.

${ }^{54}$ DCLA, Archives des Royal Dublin Fusiliers, «Collection Monica Roberts », vol. I, RDFA/001/01, lettre de Joseph Clarke à Monica Roberts, 11 mai 1916.

${ }^{55}$ LUL, Collection Liddle, GS 0741, lettre du lieutenant Hemphill à ses parents, 29 avril 1916.

${ }^{56}$ Idem.

${ }^{57}$ The Incinerator, vol. II, juin 1916, p. 19.
} 
«3430 hommes et 79 femmes ${ }^{58} »$ emprisonnés n'exécutent qu'une dizaine de rebelles. La clémence du gouvernement britannique à l'égard des rebelles consterne les partisans unionistes qui enragent de constater que la grande majorité des rebelles s'en sort avec des peines d'emprisonnement. Ce qui démoralise les hommes de la $36^{\text {ème }}$ division, c'est la dichotomie entre le traitement modéré infligé aux rebelles et la discipline militaire à laquelle ils doivent se plier. Qu'importe leur loyauté envers l'Empire, tout manquement au devoir au front est « traité sans ménagement ${ }^{59} »$.

L'impact de l'insurrection nationale se perçoit aussi sur le moral des autres combattants de l'armée britannique. Le fiasco militaire irlandais bouleverse également les autres soldats du Corps Expéditionnaire qui condamnent unanimement l'insurrection. Élargir l'étude des répercussions du soulèvement aux combattants anglais, écossais et gallois permet d'affirmer que la rébellion ne concerne pas que les combattants irlandais de l'armée britannique.

Le Père Bernard Carey, aumônier irlandais au sein d'un bataillon de l'armée des Indes, déplore le déploiement de violence verbale et s'offusque de la virulence d'un capitaine qui frappe d'anathème ces «foutus irlandais » et se demande «s'il ne faudrait pas envoyer un autre Cromwell afin de les réduire en miettes ${ }^{60} »$. Les nouvelles du soulèvement consternent beaucoup d'officiers britanniques qui ne reculent pas devant la diatribe. Les gradés attendent avec impatience que le gouvernement exécute les «émeutiers dublinois ${ }^{61}$ ». Il faut absolument «écraser les Sinn Féiners une fois pour toute ${ }^{62}$ »s'emporte le sous-lieutenant Goodwin. Qu'attendent-ils pour «abattre tous ces types ${ }^{63}$ ?» s'impatiente un lieutenant du $16^{\text {ème }}$ bataillon des Lancers. Les officiers britanniques craignent pour la sécurité du RoyaumeUni. Fomenter une rébellion pendant le conflit, c'est déstabiliser l'Empire et faciliter une victoire allemande. Les gradés n'hésitent d'ailleurs pas à évoquer une alliance entre les rebelles irlandais et les autorités allemandes. Pour Frank Day qui se trouve en Egypte lorsqu'éclate l'insurrection, le soulèvement a été financé sans aucun doute «par de l'argent allemand $^{64}$ ». C'est avec mépris que le capitaine du $7^{\text {ème }}$ bataillon du King's Shropshire Light Infantry dénonce ce « coup de couteau dans le $\operatorname{dos}^{65} »$. L'indignation ressort aussi des propos d'un soldat gallois qui s'estime « poignardé dans le dos ${ }^{66}$ » par les rebelles irlandais. Tout cela explique pourquoi les gradés et leurs hommes accueillent les nouvelles des exécutions des rebelles par «des cris de joie ${ }^{67} »$. Fomenter une rébellion alors même que l'Empire britannique livre une lutte acharnée contre l'impérialisme allemand révolte les combattants britanniques.

À en juger par les fonds d'archives disponibles, très peu d'officiers et de soldats britanniques mentionnent par écrit le soulèvement irlandais. Toutefois, à travers les quelques témoignages recueillis, un double parallèle s'établit entre le comportement des militaires de métier irlandais du Corps Expéditionnaire britannique, la réaction des engagés volontaires de la division d'Ulster et les propos des autres militaire de carrière. Les combattants de la $36^{\text {ème }}$ division nourrissent la même rancœur que les officiers et soldats britanniques. La violence de

\footnotetext{
${ }^{58}$ Archives Nationales de Londres, CO 903/19, dossier 2, « Informations des Services Secrets, 1916 », p. 35.

${ }^{59}$ The Incinerator, vol. II, juin 1916, p. 19.

${ }^{60}$ Bernard Carey, Leaves From The Diary, op. cit., p. 45

${ }^{61}$ LUL, Collection Liddle, GS 1066, journal de guerre du capitaine Mason, 27 avril 1916.

${ }^{62}$ Ibidem, GS 0644, lettre du sous-lieutenant Goodwin à sa mère, 26 avril 1916.

${ }^{63}$ Ibidem, GS 1082, lettre du lieutenant Alan Mead à sa mère, $1^{\mathrm{er}}$ mai 1916.

${ }^{64}$ Ibidem, EP 015, lettre de Frank Doughty Day à sa mère, 2 juin 1916.

${ }^{65}$ Ibidem, H.30, capitaine Hughes-Hallett, « Recollections, 1914-1918 », p. 2.

${ }^{66}$ Ibidem, GS 1064, Mason Fred Taylor, « Recollections, 1920-1921», 1972, p. 2.

${ }^{67}$ LUL, Collection Liddle, GS 0572, lettre de Sir Harold Howitt au capitaine Foster, 7ème bataillon des Sherwood Foresters, 14 mai 1916.
} 
leurs propos est d'ailleurs tout aussi forte que celle des combattants anglais, écossais ou gallois.

\section{Le questionnement identitaire des combattants nationalistes irlandais}

Alors que les comportements des hommes de la $36^{\text {ème }}$ division et des unités irlandaises du Corps Expéditionnaire britannique se recoupent, les réactions des engagés volontaires de la $16^{\text {ème }}$ division apparaissent plus ambigües. Composée en grande majorité de volontaires irlandais nationalistes partisans d'une nation irlandaise, la $16^{\text {ème }}$ division se trouve dans le nord de la France lorsqu'éclate l'insurrection de Dublin.

Certains combattants de la $16^{\text {ème }}$ division éprouvent beaucoup de ressentiment lorsqu'ils apprennent qu'une rébellion vient d'éclater en Irlande. Le capitaine Stephen Gwynn, des Connaught Rangers, se souvient de l'abattement des soldats : «Je n'oublierai jamais l'indignation des hommes. Ils avaient le sentiment d'avoir été poignardés dans le $\operatorname{dos}^{68}{ }$. Les soldats de cette unité crient à la trahison et maudissent les auteurs de la révolte. Le gradé irlandais, fervent nationaliste et partisan d'une république irlandaise, constate le traumatisme infligé par l'insurrection. Son témoignage montre de quelle manière les engagés volontaires nationalistes, défenseurs d'une nation irlandaise indépendante, accueillent les nouvelles irlandaises. L'événement atteint le moral des Connaught Rangers lesquels, impuissants, se morfondent. Nationaliste de Strabane, le lieutenant Edward Gallager du $7^{\text {ème }}$ bataillon des Royal Inniskilling Fusiliers déplore lui aussi «cette terrible rébellion » et cette «pauvre vielle Irlande, une nouvelle fois trahie ${ }^{69} »$. En ce sens, certains engagés de la $16^{\text {ème }}$ division se sentent «poignardé dans le dos ${ }^{70}$ » tout comme les engagés de la $36^{\text {ème }}$ division d'Ulster et les autres unités irlandaises de l'armée britannique.

Nourrir des aspirations nationalistes n'induit pas forcément une acceptation de l'insurrection nationale. Les événements qui se déroulent au pays infligent leur lot de souffrances et déstabilisent les combattants de la $16^{\text {ème }}$ division qui condamnent le soulèvement.

Le sentiment de révolte des engagés tient aussi aux nombreuses pertes déplorées suite à une attaque au gaz, le 27 avril 1916, peu de temps après leur arrivée en France. Ce jour-là, dans le secteur d'Hulluch, au nord de la France entre La Bassée et Lens, les soldats de la $16^{\text {ème }}$ division, abrités dans leur tranchée, serrés les uns contre les autres, fusil en main, subissent les tirs nourris de l'artillerie allemande. Bientôt, un épais «nuage jaune rampant à ras du $\mathrm{sol}^{71}$ » en provenance des lignes allemandes se propage vers les tranchées et s'y engouffre insidieusement. Les gaz parviennent jusqu'aux positions du $7^{\text {ème }}$ bataillon des Leinsters, en réserve avec la $47^{\text {ème }}$ brigade. Au petit matin du 29 avril, alors même que les insurgés irlandais déposent les armes en Irlande, des corps irlandais gisaient, eux, dans le No Man's Land. Des centaines de soldats étendus sur la ligne de feu agonisaient en "vomissant une épaisse écume verdâtre ${ }^{72} \gg$. Après les attaques, la division déplorait la perte de 2128 hommes dont 538 tués $^{73}$.

\footnotetext{
${ }^{68}$ Stephen Gwynn, John Redmond's Last Years, London, 1919, p. 230.

${ }^{69}$ Belfast Evening Telegraph, 6 mai 1916, in Jane Leonard, 'The Reaction of Irish Officers in the British Army to the Easter Rising of 1916', in Cecil Hugh \& Peter Liddle (eds.), Facing Armageddon, op. cit., p. 263.

${ }^{70}$ Stephen Gwynn, John Redmond's, op. cit., p. 230.

${ }^{71}$ IWM, 67/42/1, journal de guerre du capitaine Staniforth, « Kitchener's soldier, 1914-1918 », p. 152.

${ }^{72}$ Ibidem, 80/25/1, journal de guerre du lieutenant-colonel Lyon, p. 60.

73 Terence Denman, Ireland's Unknown Soldiers: the $16^{\text {th }}$ Division in the Great War, Dublin, Irish Academic Press, 1992, p. 143.
} 
La consternation des rescapés de la $16^{\text {ème }}$ division tient également à ce contexte. Pourtant, si les fidèles d'une nation irlandaise indépendante condamnent la rébellion, ils condamnent avec autant d'acharnement les exécutions des rebelles.

Internés dans un camp en Allemagne, quelques prisonniers du $8^{\text {ème }}$ bataillon des Royal Irish Fusiliers (16 ${ }^{\text {ème }}$ division) confient à leurs gardiens leur intention « de déserter le moment $v^{2} u^{74} »$. Les captifs s'agitent et cherchent à rejoindre le pays mais aucun ne met à exécution son plan «par peur des implications ${ }^{75}$ ». Les autorités allemandes remarquent que les prisonniers n'osent pas déserter. Patrick McMahon, admet que malgré les événements à Dublin «[les éprouvés] n'avaient aucune alternative si ce n'est continuer à combattre ${ }^{76}$ ». Partir rejoindre les rebelles irlandais, c'est s'exposer à la peine capitale, menace suffisamment dissuasive pour réprimer les velléités de fuite des captifs. Même si certains prisonniers de guerre s'insurgent contre les exécutions des meneurs, il leur est impossible de renier l'uniforme britannique. Par ailleurs, il ne s'agit pas simplement de déserter mais aussi d'abandonner leurs frères d'armes. Déserter ce serait «sortir du groupe, sortir du rang ${ }^{77}$ ». C'est aussi par solidarité avec leurs camarades que les hommes renoncent à commettre l'irréparable : «renoncer à finir la guerre, c'est-à-dire à la gagner, aurait été comme une injure proférée envers les camarades déjà tombés ${ }^{78} »$.

Leur engagement auprès de la Couronne britannique n'implique pas forcément une fidélité inconditionnelle ; la loyauté envers le gouvernement britannique se trouve limitée par la sympathie qu'éprouvent plusieurs engagés volontaires pour les « $\operatorname{martyrs}^{79}$ » des Pâques Sanglantes.

La question du châtiment infligé aux rebelles «devient très vite une question politique $^{80}$ ». Ce sont surtout les quatorze exécutions théâtralisées, « une par jour sur deux semaines $^{81}$ » qui suscitent l'indignation tant chez certains engagés volontaires de la $16^{\text {ème }}$ division que chez quelques combattants irlandais du Corps Expéditionnaire britannique. En effet, même s'il déplore l'agitation, Harry Loughlin, soldat de métier irlandais d'une unité du Corps Expéditionnaire britannique, «ne cautionne pas le traitement des Sinn Féiners ${ }^{82}$ ».

Engagé en 1912 dans l'armée britannique, John Lucy résume à lui seul l'amère incompréhension qui s'empare de plusieurs soldats de métier irlandais. Lors des «Pâques Sanglantes », le militaire de carrière du $2^{\text {ème }}$ bataillon des Royal Irish Rifles se trouve à l'hôpital de Dublin où il est soigné pour «neurasthénie ${ }^{83}$ ». Pourtant, alors que ses frères d'armes qui se trouvent en convalescence avec lui affichent «peu de sympathie pour les rebelles $^{84}$ », ils désapprouvent les exécutions des meneurs. Le sous-officier ressent lui aussi « une colère froide ${ }^{85}$ » en apprenant que des citoyens irlandais tombent sous les balles britanniques à Dublin. Dans son témoignage de guerre, le sergent écrit avec amertume :

\footnotetext{
${ }^{74}$ Christopher Duffy, Through Germany Eyes: the British and the Somme 1916, London, Weidenfeld \& Nicholson, 2006, p. 102.

${ }^{75}$ Idem.

${ }^{76}$ Ibidem, p. 103.

${ }^{77}$ Frédéric Rousseau, « Autre point de vue, autre regards. Pour une histoire européenne de la Grande Guerre », in Jules Maurin, Jean-Charles Jauffret (dir.), La Grande Guerre 1914-1918: 80 ans d'historiographie et de représentations: colloque international, Montpellier, 20-21 novembre 1998, Montpellier, Université Paul Valery, 2002, p. 411.

${ }^{78}$ François Cochet, Survivre au front, op. cit., p. 226.

${ }^{79}$ Bernard Carey, Leaves From The Diary, op. cit., p. 45.

${ }^{80}$ Neville Macready, Annals of an active life, vol. I, London, Hutchinson, 1924, p. 241.

${ }^{81}$ Francis Vane, Agin the Gouvernments: memories and adventures, London, Sampson Low, 1928, p. 271.

82 DCLA, Archives des Royal Dublin Fusiliers, «Collection Monica Roberts », vol. I, RDFA/001/02, lettre d'Harry Loughlin à Monica Roberts, 17 juin 1917.

${ }^{83}$ John Lucy, There's a Devil in the Drum, England, Naval and Military Press, 1992, p. 350.

${ }^{84}$ Ibidem, p. 352.

${ }^{85}$ Idem.
} 
Mes frères d'armes n'avaient pas beaucoup de sympathie pour les rebelles, mais ils en eurent marre quand ils apprirent les exécutions des meneurs. Je ressentis de la colère froide, et j'aurais préféré voir tout l'empire britannique brûler en enfer que d'entendre dire qu'un Irlandais avait été tué dans son propre pays par un intrus ${ }^{86}$.

La réaction des blessés irlandais interpelle également. Les nouvelles des exécutions provoquent rapidement une vive colère chez les militaires de métier du $2^{\text {ème }}$ bataillon des Royal Irish Rifles. Bientôt la répression entraîne de la compassion pour les insurgés. Les soldats britanniques qui exécutent les rebelles à Dublin deviennent des intrus frappés d'anathème. John Lucy utilise d'ailleurs le mot «tué » alors qu'il s'agit en réalité d'exécutions. Le combattant du Corps Expéditionnaire britannique remet donc en question la légitimité des soldats britanniques à mater ainsi la rébellion. Par ailleurs, le fait qu'il qualifie l'armée britannique d' «intrus » suggère que le soldat de métier nourrit des aspirations nationalistes. La présence même de soldats britanniques dans l'île lui paraît contestable. Comble d'ironie, lui qui aimerait voir « l'empire britannique brûler en enfer ${ }^{87}$ » fait partie de l'armée d' "intrus » qui écrase l'insurrection. Un aumônier catholique irlandais, posté en Égypte lors de l'insurrection, maudit lui aussi «l'escouade de barbares anglais ${ }^{88}$ » chargés de cribler de plomb les meneurs de la révolte.

La sévérité de la répression pousse même certains combattants comme Gibbon Monk à se demander s'ils combattent dans « la bonne armée ${ }^{89}$ ». L'engagé volontaire remet en cause sa participation au conflit auprès des Britanniques. Tom Barry qui, au moment de la rébellion, combat en Mésopotamie, réprouve lui aussi la mise à mort des meneurs : "Un sacré réveil, des fusils tiraient sur des gens de ma propre race, pointés par des soldats appartenant à l'armée dans laquelle je servais ${ }^{90}$. $\gg$ Les propos du combattant montrent qu'il s'identifie aux rebelles. La «race » à laquelle il appartient c'est celle des Irlandais insurgés. Ses propos suggèrent également une prise de conscience car la jeune recrue qu'il est semble désormais comprendre les conséquences de son engagement. Là encore, le peu de témoignages recueillis ne permet pas de généraliser la réaction des troupes irlandaises du Corps Expéditionnaire britannique face à la répression du soulèvement. Toutefois, il apparaît que certains militaires de métier irlandais du CEB désapprouvent le traitement fait aux rebelles.

Il convient ainsi de nuancer l'affirmation selon laquelle l'insurrection de Dublin «n'éveille aucun sentiment antibritannique que ce soit à Dublin, en dehors de la rébellion, ou en France ${ }^{91} »$. Même si nous constatons que les critiques les plus virulentes émanent des soldats de métier du Corps Expéditionnaire britannique et des engagés de la $36^{\text {ème }}$ division d'Ulster, les recrues de la $16^{\text {ème }}$ division ne sont pas les seules à s'élever contre les exécutions des meneurs. Quelques militaires de métier irlandais du Corps Expéditionnaire britannique sont en total désaccord avec la répression en Irlande.

\section{Des nouvelles inappropriées d'Irlande}

Dans d'autres unités, l'événement suscite peu de réactions et la plupart des soldats semblent afficher une indifférence sans équivoque. Le colonel Fielding, commandant du 6 ème bataillon des Connaught Rangers, n'aborde jamais le problème de l'insurrection dans les lettres qu'il adresse à sa femme ${ }^{92}$. Le Père Henry Gill, attaché au $2^{\text {ème }}$ bataillon des Royal

\footnotetext{
${ }^{86}$ John Lucy, There's a Devil, op. cit., p. 352.

${ }^{87} \mathrm{Idem}$.

${ }^{88}$ Bernard Carey, Leaves From The Diary, op. cit., p. 45.

${ }^{89}$ Gibbon Monk, Inglorious Soldier, London, Hutchinson, 1968, p. 171.

${ }^{90}$ Tom Barry, Guerilla, op. cit., p. 1.

${ }^{91}$ Douglas Gill, Dallas Golden (eds.), The Unknown, op. cit., p. 58.

${ }^{92}$ Rowland Fielding, War letters to a wife: France and Flanders 1915-1919, London, The Medicis Society1929, $382 \mathrm{p}$.
} 
Irish Rifles, écrit dans une de ses lettres que «l'événement suscite peu d'intérêt en général $^{93} »$. Son témoignage rejoint celui d'un soldat du $2^{\text {ème }}$ bataillon du Royal Irish Regiment qui confie que même si les hommes s'intéressent vaguement au soulèvement "personne ne prend la chose véritablement au sérieux ${ }^{94}$ ». Le major Frank Crozier relève qu'en général ses hommes du $9^{\text {ème }}$ bataillon des Royal Irish Rifles n'y accordent aucune attention ${ }^{95}$. Tom Kettle, du $9^{\text {ème }}$ bataillon des Royal Dublin Fusiliers, admet d'ailleurs que le soulèvement «l'ennuie quelque peu ${ }^{96} »$. L'indifférence affichée s'explique par l'omniprésence du danger auquel sont confrontés les combattants. En effet, les horreurs de la guerre « rendent les nouvelles du pays irréelles et particulièrement inappropriées ${ }^{97}$ ». C'est ce qui peut expliquer pourquoi de nombreux carnets de soldats irlandais ne contiennent pas d'allusions au soulèvement. La préoccupation essentielle est de sortir vivant du conflit. À la virulence de certains soldats irlandais s'oppose une indifférence qui peut surprendre mais qui constitue une des réalités du conflit.

Le silence des sources écrites peut également s'expliquer par une absence d'information claire. Les nouvelles de la rébellion ne parviennent pas forcément à tous les soldats et officiers irlandais. La plupart des mémoires des combattants irlandais disponibles à l'Imperial War Museum de Londres ne font jamais référence au soulèvement. Même constat pour les correspondances des combattants britanniques conservées à la Bibliothèque universitaire de l'Université de Leeds. Très peu de témoignages évoquent l'insurrection du 24 avril 1916. Même en imaginant que les nouvelles mettent un certain temps à parvenir à certaines unités irlandaises et britanniques, nous sommes bien obligés de constater, après une lecture attentive, que les carnets de guerre des soldats et officiers ne comportent aucune référence concrète à l'insurrection du 24 avril 1916. Les combattants irlandais et les autres militaires de l'armée britannique qui s'épanchent sur la rébellion restent très minoritaires.

Par ailleurs, une analyse attentive des témoignages recueillis révèle que les autorités britanniques filtrent les lettres que les combattants des Royal Dublin Fusiliers envoient à leurs marraines de guerre. Le corpus documentaire sollicité révèle également que la plupart des témoignages condamnent sans équivoque l'insurrection. Les lettres soulignent une fidélité absolue envers la Couronne britannique. Les correspondances conservées à la Bibliothèque universitaire de Leeds vont dans le même sens. En revanche, les quelques témoignages dans lesquels les combattants irlandais expriment de l'empathie pour les rebelles sont des mémoires écrites et publiées après le conflit. Les propos virulents des engagés volontaires nationalistes de la $16^{\text {ème }}$ division et des militaires de carrière irlandais du Corps Expéditionnaire britannique contre la répression ne sont retranscrits que dans leurs mémoires de guerre publiées après leur démobilisation. C'est d'ailleurs uniquement sur la foi de quelques gardes allemands qu'il est possible de conclure à une volonté de quelques captifs irlandais de partir combattre auprès des rebelles. Difficile cependant de croire que seuls les fidèles de la Couronne s'épanchent et confient à leurs familles leur ressenti suite au soulèvement. L'état actuel de nos recherches nous amène à penser que la censure a très certainement filtré les lettres des combattants irlandais confiant une quelconque sympathie pour les insurgés.

\footnotetext{
${ }^{93}$ JAD, Henry Gill, « As Seen by a Chaplain with the $2^{\text {nd }}$ battalion, Royal Irish Rifles », 80.

${ }^{94}$ IWM, P262, journal de guerre d'Anthony Brennan, p. 7.

${ }^{95}$ Frank Crozier, Impressions and Recollections, London, 1930, p. 56.

96 Timothy Bowman, The Irish regiments in the Great War: discipline and morale, Manchester, Manchester University Presse, 2004, p. 128.

${ }^{97}$ Francis Law, A Man At Arms, London, Collins, 1983, p. 69.
} 
Croiser les réactions des différentes unités britanniques suggère que les combattants de la $36^{\text {ème }}$ division et les militaires de métier des unités irlandaises du Corps Expéditionnaire britannique fustigent l'insurrection de Dublin et se réjouissent des exécutions des rebelles, tandis que la répression de la révolte provoque des réactions contrastées au sein des unités de la $16^{\text {ème }}$ division irlandaise. Certains soldats en poste en Irlande pendant le soulèvement choisissent de désobéir et de déposer les armes, quelques-uns (dans de rares cas semble-t-il) vont jusqu'à combattre auprès des rebelles. En ce sens, l'engagement dans l'armée britannique pendant le Premier Conflit mondial n'induit pas nécessairement une loyauté inconditionnelle envers la Couronne. Sans chercher ici à généraliser, les écrits de certains combattants irlandais au front suggèrent que le sort des rebelles importe plus que la sécurité de l'Irlande. Leurs propos révèlent une identification sans équivoque avec les insurgés. Même minoritaires, les témoignages de ces soldats appuient nos conclusions selon lesquelles il existe une diversité d'opinion dans les rangs des unités irlandaises de l'armée britannique. Les réactions des combattants irlandais permettent ainsi d'apprécier leurs prises de position politiques et idéologiques et nous invitent à ne plus voir les unités irlandaises comme un groupe homogène. L'insurrection du 24 avril 1916 expose les différentes typologies de soldats et officiers irlandais composant les divisions de l'armée britannique pendant le Premier Conflit mondial.

Au-delà même d'une étude strictement limitée à l'impact d'un soulèvement national sur les troupes irlandaises engagées dans un conflit mondial, cet article a cherché à mettre en avant la réaction d'autres combattants de l'armée britannique et à établir un parallèle entre l'indignation des engagés volontaires de la $36^{\text {ème }}$ division d'Ulster et la consternation de certains militaires de métier britanniques. Là encore, les recherches que nous avons menées ne permettent pas de conclure à une réaction unanime des autres combattants de l'armée britannique suite au soulèvement du 24 avril 1916, mais il ressort pourtant que quelques-uns d'entre eux s'insurgent contre les événements en Irlande. Il semblait ici nécessaire de ne plus envisager les nouvelles de l'arrière uniquement comme une source de soutien moral et de souligner à quel point la situation politique en Irlande a fragilisé le moral des combattants britanniques au front. 